\title{
28 Research Square \\ Role of pre-transplant corneal injective anti VEGF treatment in high risk transplantation corneas
}

\section{Romina Fasciani}

Policlinico A Gemelli: Policlinico Universitario Agostino Gemelli

Emanuele crincoli ( $\nabla$ emanuelecrincoli1@gmail.com )

University Hospital Agostino Gemelli: Policlinico Universitario Agostino Gemelli https://orcid.org/00000001-9996-9871

\section{Luigi Mosca}

Policlinico A Gemelli: Policlinico Universitario Agostino Gemelli

\section{Laura Guccione}

Policlinico A Gemelli: Policlinico Universitario Agostino Gemelli

\section{Alice Caristia}

Università Cattolica del Sacro Cuore: Universita Cattolica del Sacro Cuore

\section{Stanislao Rizzo}

Policlinico A Gemelli: Policlinico Universitario Agostino Gemelli

\section{Research Article}

Keywords: antiVEGF, corneal neovascularization, corneal transplant, pretreatment, graft rejection

Posted Date: April 20th, 2021

DOl: https://doi.org/10.21203/rs.3.rs-246503/v1

License: (c) (i) This work is licensed under a Creative Commons Attribution 4.0 International License.

Read Full License 


\section{Abstract}

Background In "high-risk" corneal graft recipients, like those with neovascularization of the cornea, the failure rate can exceed $35 \%$ at three years, the main cause being graft rejection. The main purpose of the study is to demonstrate the efficacy of pre-transplant Bevacizumab corneal injection in long term lowering of the prevalence of graft failure and rejection in high risk corneal transplantations. Methods This retrospective single-centre case-control study analyzed 37 eyes with significant corneal neovascularization and eligible for corneal transplantation at baseline. One group (BT group) included patients who were treated with a 3 months preoperative cycle of subconjunctival and/or intrastromal 5 $\mathrm{mg} / 0.2 \mathrm{ml}$ Bevacizumab injected at the limbus with a $27 \mathrm{G}$ needle. The control group (NT group) didn't receive the preoperative treatment. The main outcome was the prevalence of graft failures and rejections during the follow up period. Secondary outcomes included the prevalence of adverse events and the prevalence of patients who avoided corneal transplantation due to the treatment. Results BT group included 24 eyes with a follow up duration of $61.2 \pm 24$ months. Eight patients (33.3\%) avoided transplant surgery after the injection. In the BT group 5 cases of graft failure (20.8\%), 1 of which due to graft rejection (4\%), occurred during the follow up. The NT group registered 6 cases $(46.1 \%)$ of immune rejection of the graft and no additional graft failures from other causes. Conclusions Pre-transplant bevacizumab corneal injections are a safe and effective method to prevent graft rejection in neovascularized corneas.

\section{Introduction}

Corneas are the most commonly transplanted tissue worldwide: speaking in terms of numbers, 42642 corneal transplantations were performed in 2010 in the USA compared with 12623 solid-organ transplantations in 2008. [1] The irreversible loss of refractive quality of the corneal graft is defined as graft failure. Graft failure can be due to immune (graft rejection) or non-immune causes. [2] The avascular nature of the cornea, unique physiology of the anterior chamber recognized as Anterior Chamber Associated Immune Deviation (ACAID), low to absent expression of major histocompatibility antigens (MHC)-I and II in the cornea, quiescent corneal antigen-presenting cells, and presence of $\mathrm{T}$ cell apoptotic factors like Fas-ligand are some of the factors responsible for high survival rates of corneal allografts. [3] Despite the lowest rate of immune rejection among all the solid organ transplantation procedures in modern medicine, immune rejection remains the leading cause of corneal graft failure, accounting for over half the cases of graft decompensation. Other reasons for corneal graft failure include glaucoma (10\%), non-viral infections (10\%), endothelial cell failure ( $8 \%)$, and viral herpetic infections (7\%); the reasons for the remaining cases often go undetected.[2], [4] Nevertheless, not all the cases of immune rejection end up in a failure of the graft: overall, $30 \%$ of the transplanted corneas experience at least one episode of immune reaction and one third of these lead to eventual graft failure. [5], [6] The incidence of rejection is greatest in the first year-and-a-half following transplant but can occur up to 20 years or more after surgery. Guilbert et al reported an average keratoplasty-to-rejection time of $19.8 \pm 20.4$ months (among 299 patients who experienced a rejection episode). [7] Interestingly, not all 
the corneal transplant bear the same risk of graft rejection: we can distinguish between high risk (HR) transplantations and low risk (LR) transplantations. In uncomplicated or "low risk" penetrating keratoplasty (PK) primary grafts, the survival rate with local immune suppression has been reported to be as high as $95 \%$ at 5 years, with a rejection risk of $18 \%$ in 5 years and a $66 \%$ of reesulution of graft rejection without incurring in graft failure. [8] In contrast, in "high-risk" recipients the failure rate can easily exceed $35 \%$ at three years, with an incidence of graft rejection during the same period of $55 \%$. [9] We can conclude that the prevention of the onset of immune reactions in HR graft is a key factor to reduce the burden of graft failure and retransplantation in this group of patients. The reported prevalence of corneal graft rejection events compared to prevalence of corneal graft failure due to rejection over the past few decades indicates improvement in our ability to manage allograft rejection but also reveals that we do not sufficiently understand the process to control it completely. In this context, great attention has been paid to the detection of the factors that define an HR transplantation and to the underlying mechanisms for this increased susceptibility. Pre[10]-[12] and post[13] transplant corneal neovascularization is among the most studied risk factors for allograft rejection, not only for its statistical relevance but also for the interesting biological implications and therapeutical perspectives. Any vascularization of the recipient cornea to the graft margin has been proven to increase the rate of rejection. [14] Nevertheless, involvement of at least 2 corneal quadrants appears to be at high-risk for rejection[15] and graft failure and rejection risk increases with an increasing number of corneal quadrants affected by neovascularization before keratoplasty. [16] Other known risk factors include prior allograft failure (particularly due to rejection), recipient age $<40$ years old[17], large-diameter corneal grafts[18] , presence of iris synechiae[10], loose sutures in the graft, pre-existing eye inflammatory condition, prior use of glaucoma medications or prior glaucoma surgery. [19] The incidence of graft rejection is also largely influenced by the technique for corneal transplantation adopted. According to a 2015 metanalysis from Akanda ZZ et al[20] , PKP (penetrating keratoplasty) bears a pooled odds ratio for rejection of 3.56 (95\% Cl: 1.76-7.20) compared to anterior lamellar keratoplasty (ALK) and of 1.52 (95\% Cl: 1.00-2.32) compared to posterior lamellar procedures. In particular, Descemet Membrane Endothelial Keratoplasty (DMEK) is associated with a rejection rate of 0.2 in a six month follow-up period. [21]-[24] The refinement of the definition of HR corneal transplantation led us to prompt preoperative measures to reduce the risk of rejection in selected cases. An important practical approach consists of the elimination or reduction of host corneal stromal neovascularization. Different modalities of treatment have been suggested for direct or indirect occlusion of corneal vessels, such as steroids [25], radiation [26], cystine [27], cryotherapy [25], sulfuric acid [28], dextran(30), conjunctival recession [25], and laser treatment. To reduce corneal neovascularization, an alternative and innovative approach has recently been introduced which includes the use of agents against Vascular Endothelial Growth Factor (VEGF).

\section{Methods}

\subsection{Design of the study and procedures}

This retrospective single-centre case-control study analyses data from 37 eyes of 37 patients who received a corneal transplantation in the Ophthalmology department of Policlinico Agostino Gemelli 
University Hospital from May 2015 to March 2018. The study was approved by the Catholic University/Fondazione Policlinico A. Gemelli IRCCS Institutional Ethics Committee. For each patient informed consent was collected in conformity to the declaration of Helsinki. All the authors reviewed the manuscript and vouch for the accuracy and completeness of the data and for the adherence of the study to the protocol.

The main purpose of the study was to demonstrate the efficacy of pre-transplant Bevacizumab corneal injection in long term lowering of the prevalence of graft failure and graft rejection in high risk corneal transplantations, while bearing no significant burden of adjunctive adverse effects. Moreover, minor purpose of this study was to describe the morphological and functional effect of injective Bevacizumab treatment on neovascularized corneas eligible for transplant. We chose as primary outcome indexes for this purpose the prevalence of graft rejections and failures during the follow up period. Secondary outcomes included the prevalence of adverse events and the prevalence of patients who avoided corneal transplantation due to the anti-VEGF pretreatment.

All eyes included in the study showed significant corneal neovascularization ( superficial and deep vascularization with at least $1 / 4$ of corneal surface involvement) and were eligible for corneal transplantation at baseline. Exclusion criteria included: postoperative evidence of loose sutures in the graft, pre-existing eye inflammatory condition, prior use of glaucoma medications or prior glaucoma surgery, age $\leq 18$ years old. The following were considered potential confounders and analyzed by consequence: the presence of $\geq 2 / 4$ corneal quadrants involved, presence of iris synechiae, diameter of corneal graft, age $<40$ years old, type of corneal transplantation.

One group (BT group) included patients who were treated with subconjunctival and/or intrastromal 5 mg/0.2 ml Bevacizumab (Avastin_; Genetech, Inc., South San Francisco CA, USA) injected at the limbus by means of a $27 \mathrm{G}$ needle. The site of injection was the closest possible to major caliber pathologic vessels. Topical anesthesia with benoxinate and ocular and periocular disinfection with povidone iodine $5 \%$ solution were performed before the injection. Subconjunctival injection was preferred in case of four quadrants $\mathrm{CN}$ involvement and the entire dose was equally distributed in all quadrants, adjacent to the limbus. Intrastromal and subconjunctival injections were both performed in the presence of main stromal vessels: we injected half dose into the stroma at the origin of major feeder vessel and the other half dose into the adjacent conjunctiva. A cycle of three Bevacizumab injections was performed monthly by the same surgeon prior to surgery. The adverse effects attributed to the Bevacizumab treatment were recorded. A control group (NT group) of patients who didn't receive injective Bevacizumab treatment prior to transplant surgery was assessed. Both groups underwent either DALK or PK surgery after the preliminary phase in case of persistent eligibility for corneal transplant. Both groups underwent the same post-operative medication, which included:

- $4 \mathrm{mg}$ Betamethasone subtenonian injection at the end of the surgery

- $25 \mathrm{mg}$ oral Prednisone per day for 1 month (subsequently tapered gradually to $5 \mathrm{mg}$ per day), 
- Dexamethasone eyedrops tapered during the course of a month, subsequently replaced by Fluorometholone eyedrops 3 times per day for 1 year

All follow-up visits were performed by the same physician and included photographic documentation of the anterior segment status. Rejection was detected according to the referred symptoms and concurrent signs, analyzed by means of a slit lamp examination. In case of rejection patients were treated with one 4 $\mathrm{mg}$ subtenonian Betamethasone injection weekly for 3 weeks, Dexamethasone eyedrops for 2 months and $25 \mathrm{mg} /$ die oral Dexamethasone for 1 month. Irreversible graft opacities or edema were considered as treatment failure. Diagnosis of rejection was only be made in grafts that have remained clear for at least 2 weeks following surgery.

\subsection{Statistical analysis}

Sample size calculation was performed using G*power 3.1.9.7 software setting an alfa error of 0.05 and a power of the study of $80 \%$. Statistical analysis was performed using SPSS software (IBM SPSS Statistics 26.0). Chi ${ }^{2}$ test was used to compare BT group and NT group as concerns the prevalence of graft rejection anf failure during the follow up time. Fisher's exact test was used when appropriate. Age, follow up duration and diameter of the corneal graft were considered as continuous quantitative variables and were described using mean value and standard deviation in case they were normally distributed and median value and interquartile range (IQR) in case of divergence from normal distribution. Normality test was executed according to Shapiro-Wilk method. Logistic regression analysis was performed in order to clear the results from the effect of potential confounding factors. A $p$ value $<0.05$ was considered as statistically significant.

\section{Results}

BT group included 24 eyes of 24 patients who were 11 males (45,8\%) and 13 females (54,2\%). Their mean age was $54 \pm 5,2$ years. Mean total duration of the follow up from the BT GROUP was $61.2 \pm 24$ months (ranging from 24 to 108 months). Neovascularization in eyes from the BT GROUP was due to different eziologies:

- 5 cases of non herpetic post-infective leukomas

- 11 cases of post- herpetic leukomas

- 3 cases of traumatic leukomas

- 5 cases of corneal graft rejection (previous corneal transplant)

The control group (NT group) included 13 eyes from 13 patients. The mean age of the patients was $56 \pm$ 7,1 years and they were 6 males (46,2\%) and 7 females (53,8\%). NT group mean total duration of the follow-up was $26.1 \pm 5.7$ months (ranging from 16 to 36 months). The causes of neovascularization included: 
- 6 cases of post-herpetic leukomas

- 7 cases of corneal graft rejection (previous corneal transplantation)

All the patients from the BT group showed a significant reduction of vessel congestion and milder signs of ocular surface inflammation since the first Bevacizumab injection. The most evident effect of the treatment was the reduction of the caliber of the vessels, in particular the more superficial, thinner and newer ones. The extension of the neovascularized corneal surface encountered a variable degree of reduction, with better results in postherpetic leucomas. No patient showed adjunctive local or systemic adverse effects due to the treatment with Bevacizumab.

Only 16 patients from the BT group underwent transplant surgery after the preliminary treatment with injective Bevacizumab: the 8 remaining patients (33.3\%) avoided transplant surgery due to sufficient remission of the pathological process. Seven (7) DALK surgeries and 9 PK surgeries were perfomed on the remaining 16 patients from the BT group. In the NT group (13 patients) 6 DALK and 7 PK surgeries were carried out. The keratoplasty procedures were performed without intraoperative and postoperative complications in both groups.

In the BT group only 1 case of rejection (4\%) occurred during the follow up. The timing of the rejection was 24 months after the surgery. The BT group registered 5 cases of graft failure (20.8\%) during the follow up (Table 1)

Table 1. Cases of graft failure among the patients in BT group. DALK = Deep anterior lamellar keratoplasty; DSAEK $=$ Descemet Stripping Automated Endothelial Keratoplasty; IOP = intraocular pressure; PK = Penetrating keratoplasty;

\begin{tabular}{llll}
\hline $\begin{array}{l}\text { Graft failure } \\
\text { cases }\end{array}$ & Cause of the failure & $\begin{array}{l}\text { Surgical technique of the first } \\
\text { transplant }\end{array}$ & $\begin{array}{l}\text { Sugical technique of the } \\
\text { retransplant }\end{array}$ \\
\hline $\mathbf{1}$ & Corneal abscess & DALK & PK \\
$\mathbf{2}$ & Endothelial failure (high IOP) & PK & PK \\
$\mathbf{3}$ & Endothelial failure (high IOP) & PK & PK \\
$\mathbf{4}$ & Endothelial failure (high IOP) & PK & DSAEK \\
$\mathbf{5}$ & Endothelial failure (immune & PK & PK \\
& rejection) & & \\
\hline
\end{tabular}


The remaining 11 grafts from the BT group (79.9\%) survived the follow up showing with good transparency of the central $4 \mathrm{~mm}$ (optic zone). Examples of therapeutic success from the BT group are illustrated in Figure 1.

In the NT group we identified 6 cases (46.1\%) of immune rejection of the graft: 2 of them involved corneas implanted with DALK technique while 4 cases occurred in PK implanted corneal grafts. Mean time to rejection was $3.8 \pm 1.4$ months. At the end of the follow up, no other graft failure from other causes was detected: the remaining 7 grafts (53.8\%) from the NT group made it to the end of the follow up preserving the trasparency of the central $4 \mathrm{~mm}$ of the cornea (optic zone).

Univariate analysis detected a significant difference in terms of cases of immune rejection between the two groups $(p=0.01)$. Despite the higher prevalence of graft failure in the NT group, this difference didn't turn out to be statistically relevant $(\mathrm{p}=0.43$ ). No patients showed ocular or systemic adverse events related to Bevacizumab injection during the course of the follow up. Regression analysis confirmed a statistically relevant difference as concerns cases of immune rejection between the two groups $(p=0.015)$. The results of inferential statistical analysis are summarized in Table 2.

Table 2 Comparison of the 2 study groups on general variables, study endpoints and potential confounders. DALK = Deep anterior lamellar keratoplasty; PK = Penetrating keratoplasty 


\begin{tabular}{|c|c|c|c|}
\hline Variable & BT group & NT group & $\mathbf{p}$ \\
\hline Age (years), median (IQR) & $54(5,2)$ & $56(7,1)$ & 0.88 \\
\hline \multirow[t]{2}{*}{ Sex } & $M=11 / 24(45.8 \%)$ & $M=6 / 13(46,2 \%)$ & 0.76 \\
\hline & $F=13 / 24(56,2 \%)$ & $F=7 / 13(53,8 \%)$ & \\
\hline Follow up (months) & $61.2 \pm 24$ & $26.1 \pm 5.7$ & 0.001 \\
\hline \multirow[t]{2}{*}{ Surgical technique } & DALK $=7 / 16(43,8 \%)$ & DALK $=6 / 13(46,2 \%)$ & 0.61 \\
\hline & $\mathrm{PK}=9(56,2 \%)$ & $\mathrm{PK}=7 / 13(53,8 \%)$ & \\
\hline$\geq 2 / 4$ corneal quadrants involved & $5 / 16(31,3 \%)$ & $5 / 13(38,5 \%)$ & 0.55 \\
\hline Iris synechiae & $2 / 16(12,5 \%)$ & $1 / 13(7,7 \%)$ & 0.75 \\
\hline Diameter of corneal graft (mm), median (IQR) & $7.78(1,2)$ & $7.91(1,5)$ & 0.64 \\
\hline Age $<40$ years & $1 / 16(6,3 \%)$ & $1 / 13(7,7 \%)$ & 0.81 \\
\hline Retransplants & $5 / 16(31,3 \%)$ & $7 / 13(53,8 \%)$ & 0.12 \\
\hline Immune rejections & $1 / 16(6,3 \%)$ & $6 / 13(46,2 \%)$ & 0.015 \\
\hline Time to immune rejection (months) & 24 & $3.8 \pm 1.4$ & 0.11 \\
\hline Graft failures & $5 / 16(31,3 \%)$ & $6 / 13(46,2 \%)$ & 0.43 \\
\hline Adverse events & 0 & 0 & l \\
\hline
\end{tabular}

\section{Discussion}

Nowadays, graft rejection among high risk corneal transplantations largely impairs the possibility of a stable and effective therapeutical option for these patients. The results of our study showed a clinically and statistically significant lower prevalence of immune rejections in patients who received Bevacizumab corneal injection prior to the surgery, envisaging an improvement in perspectives for neovascularized high risk corneas. It is important to notice that this result was achieved without any additional ocular or systemic adverse event. Previous studies also reported the effectiveness of subconjunctival and intrastromal bevacizumab as a preconditioning treatment to improve prognosis in high-risk corneal transplantation.[29], [30] Coherently to this, recent literature reported that subconjunctival administration 
of Aflibercept as a stand-alone measure and in combination with laser coagulation of ingrowing vessels may increase the chances of transparent transplant acceptance in high-risk keratoplasty.[31] An explanation for this positive outcome lays in the downregulating effect of anti-VEGFs towards inflammation and in the vasoconstrictive local effect that they elicite. Once again, it is critical to emphasize that the most important prognosticator of graft success is the status of the recipient bed in which the corneal graft is placed: inflamed host beds are extremely prone to rejection and the removal of this stimulus is of utmost importance.[32]

Regression of corneal opacity and neovascularization, with good restoration of visual acuity after injective treatment with Bevacizumab, allowed 8 out of 24 patients from the BT group to avoid corneal transplant. This result was particularly relevant for 4 of these 8 patients, whose neovascularization was due to rejection of a previous corneal transplant (either DALK or PK). In fact, in their case preliminary Bevacizumab injective treatment avoided corneal retransplantation, which is a major and adjunctive risk factor for corneal rejection of the newly implanted graft. A recent finding from Kuo et al.[33] also reported 3 cases of regression of corneal neovascularization that allowed to avoid corneal transplantation following long term topical bevacizumab eyedrops. A 2020 retrospective study[25] reported an incidence of complete regression of neovascularization after intrastromal bevacizumab injection in $14.2 \%$ cases. In a prospective study from S Belghmaidi et al.[34] injective bevacizumab treatment was marked by a decrease in the percentage of corneal neovascularization compared to the total corneal surface area, from $45 \%$ to $28 \%$ at Day 120 .

Our paper, didn't outline any difference in terms of burden of graft failures between the two groups. BT group failures happened in the majority of the cases in grafts implanted with the PK surgical technique. A possible explanation is that lamellar transplants are attempted in corneas with subtotal compromission of the corneal recipient tissue, thus reflecting a less critical starting condition of these patients. The same prevalence of prior graft failures (retransplants) in the two groups excludes the contribution of this major risk factor to the detected differences in terms of graft rejections. Also no contribution from any other of the confounding factors analyzed was detected as concerns the primary outcome.

The Bowman Club survey in the United Kingdom reported that $53 \%$ of the members routinely commenced preoperative treatment (monotherapy or a combination of 2 or more agents) in high risk cases. Topical dexamethasone (3 to 6 hourly, duration unspecified) was used by $33 \%$ of surgeons, oral prednisolone (40 to $80 \mathrm{mg}$ for 2 to 7 days) by $22 \%$, oral cyclosporine A ( 3 to $8 \mathrm{mg} / \mathrm{kg}$, duration unspecified) by $14 \%$, and single-dose intravenous methylprednisolone by $14 \%$.[3], [35] In a rat study from Claerhout et al.[36], a preoperative dosing schedule of systemic cyclosporine A followed by postoperative treatment had no advantage in graft rejection rate over a solely postoperative treatment regimen. By contrast, in an animal model study from Kim et al.[37], the combined preoperative and postoperative administration of corticosteroid injections gave an advantage in terms of rate of graft rejection over the use of postoperative injections alone. Attempts have also been made to reduce neovascularization in candidates for possible corneal transplant without analyzing the effects on subsequent rate of graft rejection. In a study[34] with 112 participants receiving topical corticosteroids, clinical course was 
marked by a decrease in the percentage of corneal neovascularization compared to the total corneal surface area, from $45 \%$ (between 16 and $82 \%$ ) to $28 \%$ (between 0 and $69 \%$ ) at Day 120, despite the absence of statistically significant improvement in visual acuity. Among those patients, $29.4 \%$ also received subconjunctival injections of bevacizumab and $22.32 \%$ intrastromal bevacizumab injections. As concerns animal studies, endotoxin-induced corneal neovascularization in rabbits was treated with betacyclodextrin tetradecasulfate coadministered with either: hydrocortisone, tetrahydrocortisol-S, or 6-alphafluoro-17,21-dihydroxy-16-beta-methyl-pregna-4,9(11),diene,3,20-dione. When optimal ratios of steroid and cyclodextrin were used, neovascularization was reduced to $13 \%, 26 \%$, and $28 \%$ of untreated controls for the three steroids, respectively.[28] In another rabbit model, focal X-ray irradiation to the corneal region suppressed induced corneal angiogenesis in a dose-dependent manner.[26] Lastly, in vivo analysis of angiogenesis demonstrated that treatment with the cysteine peptidase inhibitor E64 caused a reduction in neovascularization.[27] As concerns physical methods, laser treatment, using $577 \mathrm{~nm}$ yellow dye laser has been shown to be an effective modality for corneal vessel occlusion[38] and a long term efficacy is also achievable in fine needle diathermy before corneal grafting.

In this panorama, anti-VEGF injective treatment represents a promising and validated therapeutical perspective. In our study, the possibility to detect a subgroup of patients that could benefit more from the treatment in object was partially impaired by the small sample size and the retrospective nature of the analysis. Nevertheless, the encouraging clinical outcome can be a stimulus to the ophthalmic community to start larger scale trials on the use of this interesting variation to the standard treatment.

\section{References}

[1] D. T. H. Tan, J. K. G. Dart, E. J. Holland, and S. Kinoshita, "Corneal transplantation," Lancet, vol. 379, no. 9827, pp. 1749-1761, May 2012, doi: 10.1016/S0140-6736(12)60437-1.

[2] Yureeda Qazi and Pedram Hamrah, "Corneal Allograft Rejection: Immunopathogenesis to Therapeutics," J Clin Cell Immunol., Nov. 2013.

[3] Di Zazzo A., Kheirkhah A., Abud T.B., Goyal S., and Dana R., "Management of high-risk corneal transplantation," Surv. Ophthalmol., no. 62, pp. 816-827, 2017.

[4] "The Australian Corneal Graft Registry. 1990 to 1992 report," Aust N Z J Ophthalmol, vol. 21, no. 2 Suppl, pp. 1-48, May 1993.

[5] A. Panda, M. Vanathi, A. Kumar, Y. Dash, and S. Priya, "Corneal graft rejection," Surv Ophthalmol, vol. 52, no. 4, pp. 375-396, Aug. 2007, doi: 10.1016/j.survophthal.2007.04.008.

[6] M. R. Dana, Y. Qian, and P. Hamrah, "Twenty-five-year panorama of corneal immunology: emerging concepts in the immunopathogenesis of microbial keratitis, peripheral ulcerative keratitis, and corneal transplant rejection," Cornea, vol. 19, no. 5, pp. 625-643, Sep. 2000, doi: 10.1097/00003226200009000-00008. 
[7] E. Guilbert, J. Bullet, O. Sandali, E. Basli, L. Laroche, and V. M. Borderie, "Long-term rejection incidence and reversibility after penetrating and lamellar keratoplasty," Am. J. Ophthalmol., vol. 155, no. 3, pp. 560-569.e2, Mar. 2013, doi: 10.1016/j.ajo.2012.09.027.

[8] J. Hjortdal, I. B. Pedersen, S. Bak-Nielsen, and A. Ivarsen, "Graft rejection and graft failure after penetrating keratoplasty or posterior lamellar keratoplasty for fuchs endothelial dystrophy," Cornea, vol. 32, no. 5, pp. e60-63, May 2013, doi: 10.1097/IC0.0b013e3182687ff3.

[9] M. C. Bartels, I. I. N. Doxiadis, T. P. Colen, and W. H. Beekhuis, "Long-term outcome in high-risk corneal transplantation and the influence of HLA-A and HLA-B matching," Cornea, vol. 22, no. 6, pp. 552556, Aug. 2003, doi: 10.1097/00003226-200308000-00013.

[10] J J Arentsen, "Corneal transplant allograft reaction: possible predisposing factors.," Trans Am Ophthalmol Soc., no. 81, pp. 361-402., 1983.

[11] J. R. Batchelor et al., "HLA matching and corneal grafting," Lancet, vol. 1, no. 7959, pp. 551-554, Mar. 1976, doi: 10.1016/s0140-6736(76)90355-x.

[12] H. J. Völker-Dieben, J. D’Amaro, and C. C. Kok-van Alphen, “Hierarchy of prognostic factors for corneal allograft survival," Aust NZ J Ophthalmol, vol. 15, no. 1, pp. 11-18, Feb. 1987, doi: 10.1111/j.1442-9071.1987.tb00300.x.

[13] Poirier $\mathrm{RH}$, “Corneal allograft reaction and its relationship to suture site neovascularization.," Ophthalmic Surg, p. 8:71-4, 1977.

[14] "Design and methods of The Collaborative Corneal Transplantation Studies. The Collaborative Corneal Transplantation Studies Research Group," Cornea, vol. 12, no. 2, pp. 93-103, Mar. 1993, doi: 10.1097/00003226-199303000-00001.

[15] Foulks GN, "Presensitization to HLA antigens in low risk keratoplasty patients.," Invest Ophthalmol Vis Sci, p. (Suppl.):227, 1987.

[16] B. Bachmann, R. S. Taylor, and C. Cursiefen, "Corneal neovascularization as a risk factor for graft failure and rejection after keratoplasty: an evidence-based meta-analysis," Ophthalmology, vol. 117, no. 7, pp. 1300-1305.e7, Jul. 2010, doi: 10.1016/j.ophtha.2010.01.039.

[17] M. G. Maguire et al., "Risk factors for corneal graft failure and rejection in the collaborative corneal transplantation studies. Collaborative Corneal Transplantation Studies Research Group," Ophthalmology, vol. 101, no. 9, pp. 1536-1547, Sep. 1994, doi: 10.1016/s0161-6420(94)31138-9.

[18] P. M. Cherry et al., "An analysis of corneal transplantation: I-graft clarity," Ann Ophthalmol, vol. 11, no. 3, pp. 461-469, Mar. 1979. 
[19] Writing Committee for the Cornea Donor Study Research Group et al., "Factors associated with corneal graft survival in the cornea donor study," JAMA Ophthalmol, vol. 133, no. 3, pp. 246-254, Mar. 2015, doi: 10.1001/jamaophthalmol.2014.3923.

[20] Zarique ZAkanda, Abdul Naeem, Elizabeth Russell, Jillian Belrose, Francie F. Si, and William G. Hodge, "Graft Rejection Rate and Graft Failure Rate of Penetrating Keratoplasty (PKP) vs Lamellar Procedures: A Systematic Review," PLoS One., p. 10(3): e0119934., 2015.

[21] Quilendrino R, "Clinical outcome of 500 consecutive cases undergoing Descemet's membrane endothelial keratoplasty," Ophthalmology, p. 122(3):464-70., 2015.

[22] Price MO, Anshu A, and Prince FW, “Descemet's stripping endothelial keratoplasty: long-term graft survival and risk factors for failure in eyes with preexisting glaucoma," Ophthalmology, p. 119(10):1982-7., 2012.

[23] I. Dapena, L. Ham, M. Netuková, J. van der Wees, and G. R. J. Melles, "Incidence of early allograft rejection after Descemet membrane endothelial keratoplasty," Cornea, vol. 30, no. 12, pp. 1341-1345, Dec. 2011, doi: 10.1097/ICO.0b013e31820d8540.

[24] C. Monnereau, M. Bruinsma, L. Ham, L. Baydoun, S. Oellerich, and G. R. J. Melles, “Endothelial cell changes as an indicator for upcoming allograft rejection following descemet membrane endothelial keratoplasty," Am. J. Ophthalmol., vol. 158, no. 3, pp. 485-495, Sep. 2014, doi:

10.1016/j.ajo.2014.05.030.

[25] D. Gupta and C. Illingworth, "Treatments for corneal neovascularization: a review," Cornea, vol. 30, no. 8, pp. 927-938, Aug. 2011, doi: 10.1097/IC0.0b013e318201405a.

[26] Miyamoto $\mathrm{H}$ et al., "Suppression of experimental corneal angiogenesis by focal X-ray irradiation," Curr Eye Res., Jun. 1999.

[27] Coppini LP et al., "Corneal angiogenesis modulation by cysteine cathepsins: In vitro and in vivo studies.," Exp Eye Res, May 2015.

[28] W. W. Li, R. Casey, E. M. Gonzalez, and J. Folkman, "Angiostatic steroids potentiated by sulfated cyclodextrins inhibit corneal neovascularization," Invest. Ophthalmol. Vis. Sci., vol. 32, no. 11, pp. 28982905, Oct. 1991.

[29] R. Fasciani, L. Mosca, M. I. Giannico, S. A. Ambrogio, and E. Balestrazzi, "Subconjunctival and/or intrastromal bevacizumab injections as preconditioning therapy to promote corneal graft survival," Int Ophthalmol, vol. 35, no. 2, pp. 221-227, Apr. 2015, doi: 10.1007/s10792-014-9938-4.

[30] P. I. Vassileva and T. G. Hergeldzhieva, "Avastin use in high risk corneal transplantation," Graefes Arch Clin Exp Ophthalmol, vol. 247, no. 12, pp. 1701-1706, Dec. 2009, doi: 10.1007/s00417-009-1170-y. 
[31] S. V. Trufanov, S. A. Malozhen, D. A. Krakhmaleva, Z. V. Surnina, E. A. Pivin, and E. A. Kasparova, "[Antiangiogenic therapy in high-risk keratoplasty]," Vestn Oftalmol, vol. 136, no. 4, pp. 11-18, 2020, doi: 10.17116/oftalma202013604111.

[32] A. Amouzegar, S. K. Chauhan, and R. Dana, "Alloimmunity and Tolerance in Corneal Transplantation," J. Immunol., vol. 196, no. 10, pp. 3983-3991, 15 2016, doi: 10.4049/jimmunol.1600251.

[33] H.-H. Kuo and E. P. Shen, "Long-term topical bevacizumab for prevention of corneal graft rejections," Eur J Ophthalmol, p. 1120672120939504, Jul. 2020, doi: 10.1177/1120672120939504.

[34] S. Belghmaidi, I. Hajji, W. Ennassiri, R. Benhaddou, T. Baha Ali, and A. Moutaouakil, "[Management of corneal neovascularization prior to corneal transplantation: Report of 112 cases]," J Fr Ophtalmol, vol. 39, no. 6, pp. 515-520, Jun. 2016, doi: 10.1016/j.jfo.2016.01.009.

[35] P. Y. P. Koay, W. H. Lee, and F. C. Figueiredo, "Opinions on risk factors and management of corneal graft rejection in the United kingdom," Cornea, vol. 24, no. 3, pp. 292-296, Apr. 2005, doi: 10.1097/01.ico.0000138841.44926.f8.

[36] I. Claerhout, H. Beele, A. Verstraete, C. Van den Broecke, and P. Kestelyn, "The effect of duration and timing of systemic cyclosporine therapy on corneal allograft survival in a rat model," Graefes Arch Clin Exp Ophthalmol, vol. 239, no. 2, pp. 152-157, Feb. 2001, doi: 10.1007/s004170000242.

[37] H. K. Kim, J. A. Choi, H. Uehara, X. Zhang, B. K. Ambati, and Y. K. Cho, "Presurgical corticosteroid treatment improves corneal transplant survival in mice," Cornea, vol. 32, no. 12, pp. 1591-1598, Dec. 2013, doi: 10.1097/ICO.0b013e31829ebb0d.

[38] J. C. Baer and C. S. Foster, "Corneal laser photocoagulation for treatment of neovascularization. Efficacy of 577 nm yellow dye laser," Ophthalmology, vol. 99, no. 2, pp. 173-179, Feb. 1992.

\section{Declarations}

Funding: The authors did not receive support from any organization for the submitted work.

Conflicts of interest: No conflicting of interest exists for any author

Ethics approval: All procedures performed in the study were in accordance with the ethical standards of the institutional and/or national research committee and with the 1964 Helsinki declaration and its later amendments or comparable ethical standards. The study has been evaluated by the Catholic University/Fondazione Policlinico A. Gemelli IRCCS Institutional Ethics Committee and deemed not to require ethics approval.

\section{Figures}



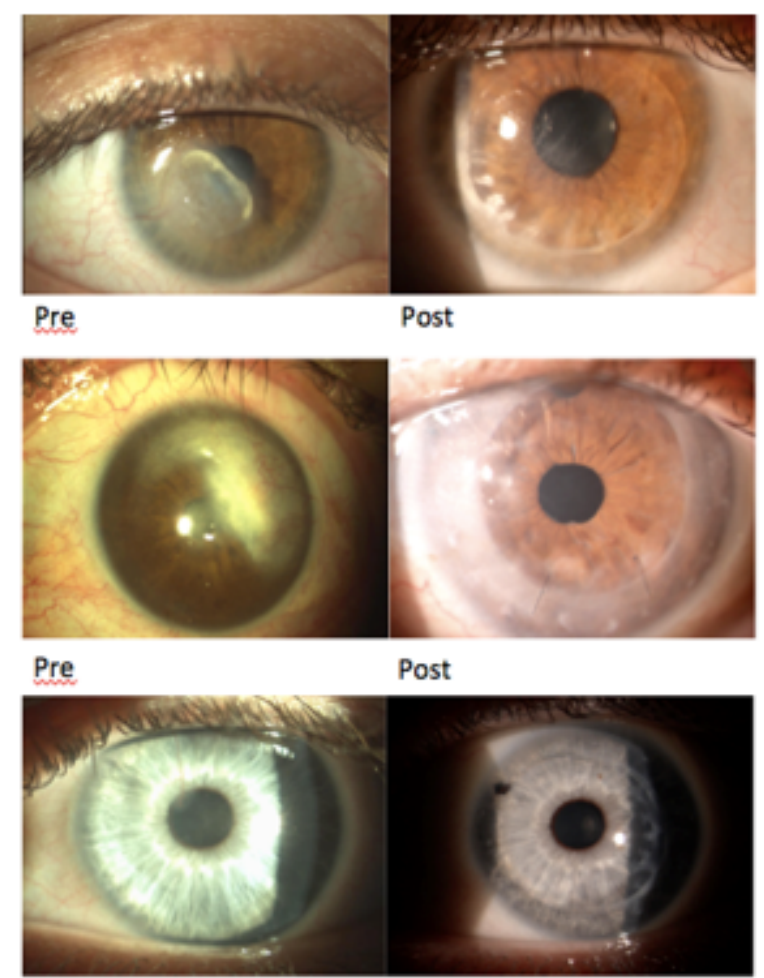

Pre

Post

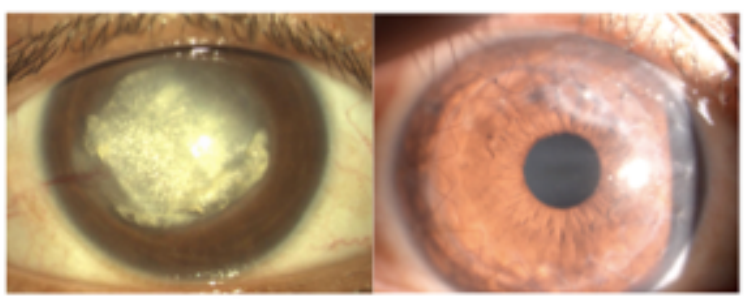

Pre
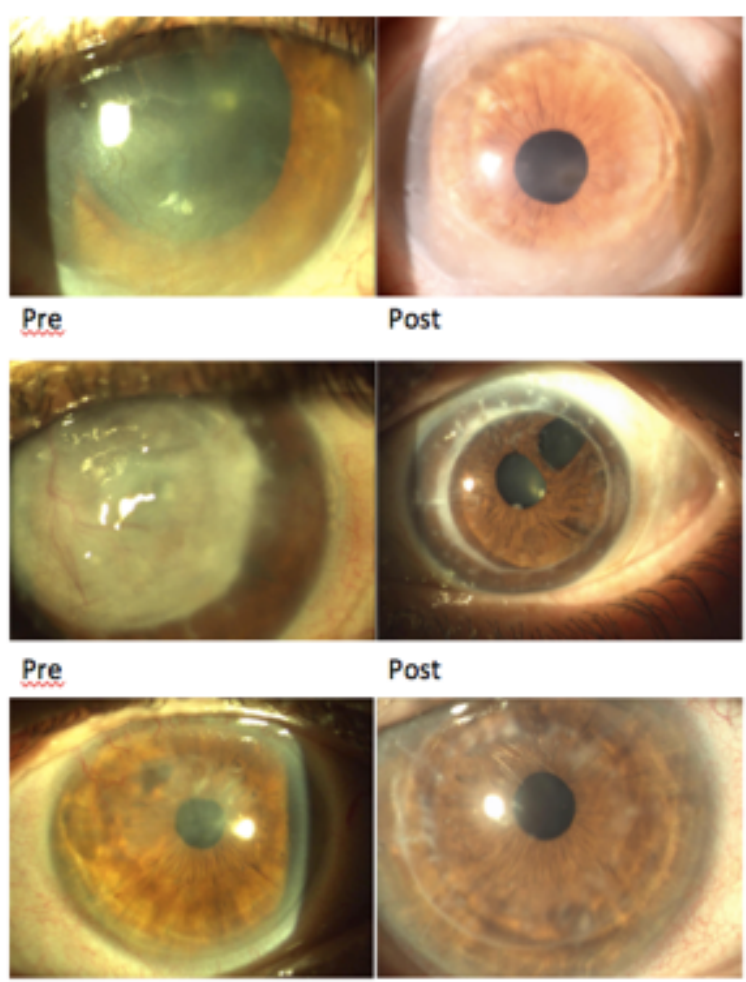

Pre

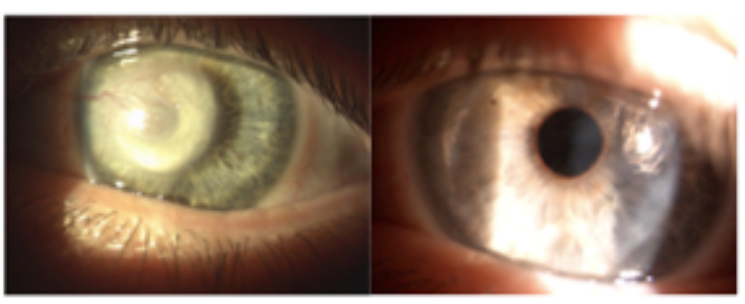

Pre

Post

\section{Figure 1}

Eight examples of therapeutic success from the BT group: each pair of anterior segment photos shows the eye of the same patient before and after anti-VEGF injection combined with corneal transplant. VEGF $=$ Vascular endothelial growth factor 